\title{
Control de un Convertidor Elevador de Voltaje AC/DC Utilizando Balance de Potencias
}

\author{
Nicolás Muñoz-Galeano, Juan B. Cano-Quintero, y Jesús M. López-Lezama \\ Fac. de Ingeniería, Depto. Ing. Eléctrica, Grupo de Manejo Eficiente de la Energía - GIMEL, \\ Universidad de Antioquia, Calle 67 No. 53-108, Oficina 19-437, Medellín-Colombia \\ (e-mail: nicolas.munoz@udea.edu.co, bernardo.cano@udea.edu.co,jmaria.lopez@udea.edu.co)
}

Recibido Sep. 1, 2015; Aceptado Oct. 28, 2015; Versión final Dic. 21, 2015, Publicado Abr. 2016

\begin{abstract}
Resumen
Este artículo propone el control de un convertidor AC/DC elevador con corrección de factor de potencia usando el balance de potencias entre la entrada y la salida. Se presenta la explicación del funcionamiento del convertidor AC/DC y se establece la secuencia de disparo del convertidor. Como principal contribución se presenta el balance de potencias para obtener la amplitud de la corriente de referencia en la entrada del convertidor que permite a su vez controlar el voltaje DC en su salida. Se utiliza un control de banda de histéresis para la corriente de entrada, permitiendo que la estrategia de control funcione con rapidez. El control en banda de histéresis propuesto funciona con rapidez y permite trabajar en un amplio rango de operación. Se presentan resultados experimentales que permiten validar el control propuesto.
\end{abstract}

Palabras clave: electrónica de potencia; convertidor elevador; factor de potencia; distorsión armónica; controlador digital de señales (DSC).

\section{Control of Step-up Voltage Converter AC/DC using Balance of Powers}

\begin{abstract}
This paper proposes the control of an AC/DC boost converter with power factor correction using input and output power balance. The operation of the AC/DC converter is presented and the firing sequence of the converter is set is established. As a main contribution, the power balance is presented to obtain the amplitude of the input reference current that allows controlling the DC voltage output. Hysteresis band control for the input current is used, allowing the quick functioning of the control strategy. The proposed hysteresis band control works quickly and allows working in a wide operating range. Experimental results which validate the proposed control are presented.
\end{abstract}

Keywords: power electronics; boost converter; power factor; harmonic distortion; digital signal controller (DSC) 


\section{INTRODUCCIÓN}

La generación de energía eléctrica normalmente se realiza mediante alternadores AC. La energía generada se transfiere a cargas que requieren suministro alterno (AC) o continuo (DC). Disponiendo de sistemas en $A C$ es posible alimentar cargas DC, para esto se utilizan convertidores AC/DC. Los convertidores AC/DC más comunes son el puente de diodos (también conocidos como rectificadores no controlados) y se usan para obtener tensión DC a partir de un sistema de distribución AC. Los rectificadores no controlados son ampliamente usados en aparatos electrónicos, por mencionar algunas aplicaciones: fuentes de alimentación conmutadas, fuentes de potencia ininterrumpidas y sistemas de carga para vehículos híbridos (Musavi et al., 2012; Jovanovic et al., 2005). Los rectificadores no controlados son fiables y fáciles de diseñar. Para regular la tensión en la salida (tensión DC), se utiliza un condensador que mantiene la tensión DC en un valor promedio constante, no permitiendo que la tensión cambie bruscamente, es decir que tenga un rizado de tensión bajo. No obstante, El uso del condensador en la salida ocasiona que los diodos del puente solo se polaricen directamente cuando la tensión DC es mayor que la tensión de la red de suministro, esto solo sucede en periodos muy cortos cerca al valor pico de la tensión de la red de suministro. Es por esto que, la corriente en la entrada es pulsante, de tal modo que se presenta distorsión armónica alta en la señal-de corriente de la red. Adicionalmente los rectificadores no controlados no permiten regular el voltaje DC en su salida.

La introducción de armónicos en la corriente de la red tiene numerosos efectos perjudiciales, dentro de los que se destacan: 1) El aumento de las pérdidas de potencia en los cables, en los transformadores y en los generadores de la red (Montano et al., 2005). 2) La aparición de corrientes por el neutro en el sistema de distribución (Muñoz et al., 2013). 3) La aparición de fallas en los dispositivos de protección (Zielichowski et al., 1999). Para mejorar la eficiencia y calidad de la potencia en la entrada y regular la tensión DC en la salida normalmente son usados convertidores elevadores AC/DC. Los convertidores elevadores son encontrados normalmente en inversores, como etapa inicial para generar un bus DC regulado.

Existen gran variedad de topologías para convertidores que permiten mejorar la calidad de suministro en las redes de distribución, una revisión de artículos que incluyen las topologías puede consultarse en (Singh et al., 2003). Las estrategias de control para convertidores elevadores buscan corregir el factor de potencia llevándolo a uno, estas estrategias normalmente son llamadas PFC (Power Factor Correction). Las estrategias PFC pretenden controlar la corriente de entrada del convertidor, de tal forma que, la forma de onda de la corriente tiene la misma forma de onda del voltaje y además están en fase (Jovanovic et al., 2005; Lim et al., 1999; Das et al., 2012).

Los artículos de la literatura técnica y que reportan estructuras de control para convertidores de potencia utilizan normalmente controladores PI (Qin et al., 2014; Lin et al., 2006; Zhang et al., 2016; Tsang et al., 2005). Dichos controladores PI requieren el conocimiento amplio del sistema debiendo linealizar y trabajar en un punto de operación. De hecho los convertidores son sistemas no lineales y ante el cambio del punto de operación pueden funcionar de forma inapropiada. La implementación del control para convertidores basados en electrónica requieren normalmente controles en cascada con un lazo de control PI de corriente interno y con un lazo de control PI de tensión externo, esto requiere que los controladores PI estén muy bien sintonizados. En contraste, el control de banda de histéresis puede trabajar en un rango amplio de operación porque no se utilizan técnicas de linealización para ajustar el punto de operación, el control de banda de histéresis se ajusta fácilmente ante los cambios del punto de operación. El control de banda de histéresis tiene como desventaja que tiene un error muy grande en estado estacionario, sin embargo este artículo propone el uso del balance de potencia entre la entrada y la salida y el cálculo de una expresión de error que permite reducir el error en estado estacionario. El control propuesto funciona satisfactoriamente, es rápido y con bajo error en estado estacionario.

Este trabajo de investigación propone el control de un convertidor AC/DC mediante del control basado en banda de histéresis y el uso del balance de potencias. La sección 1 presenta el principio de funcionamiento del convertidor AC/DC y se establece la secuencia de disparo del convertidor. Luego, en la sección 2, se describe el sistema de control propuesto basado en el balance de potencias del sistema. Se indica el procedimiento utilizado para garantizar, simultáneamente, el control sobre el voltaje de salida del convertidor, sobre el factor de potencia y la distorsión armónica de la corriente de entrada. El control propuesto permite una mayor facilidad de diseño e implementación, permitiendo el uso de sistemas digitales económicos y tiempos reducidos para el desarrollo. El sistema de control ha sido probado en una plataforma experimental. 


\section{PRINCIPIO DE FUNCIONAMIENTO Y SECUENCIA DE DISPARODELCONVERTIDOR AC/DC}

En este artículo, se ha elegido la topología de convertidor elevador PFC sin puente de (Mitchell, 1983) para implementar el control basado en el balance de potencias, ver figura 1. Esta topología presenta ventajas sobre otras topologías porque presenta mejor eficiencia y porque para su implementación requiere menos semiconductores. La bobina (L) del convertidor almacena energía para luego transferirla al capacitor (C) y a la carga $(R)$. El capacitor intercambia energía con la bobina para luego transferirla a la carga $(R)$. Se define $V_{e}$ e $I_{e}$ como el voltaje y la corriente de entrada del convertidor, mientras que se define $V_{s}$ e $I_{s}$ como el voltaje y la corriente de salida del convertidor.

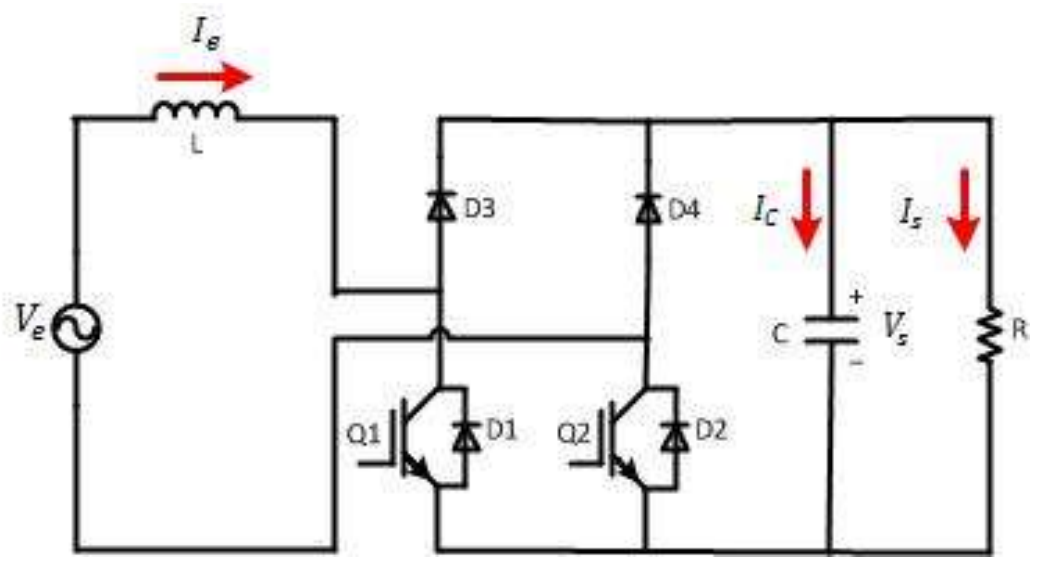

Fig 1. Circuito elevador con PFC y sin puente

Este convertidor disminuye las pérdidas de potencia al utilizar menos elementos en serie entre la salida y la entrada del circuito. Además, permite mejorar el rizado de la corriente de entrada al conectar directamente la bobina en serie con la fuente AC. La operación de los interruptores Q1 y Q2 depende de los semiciclos de la red de suministro: 1) Para el semiciclo positivo, debe operar Q1, de tal manera que Q1, D2 y D3 conforman un convertidor elevador. En el semiciclo positivo, de forma natural, D2 y D3 están cerrados debido a que se polarizan directamente; cuando el interruptor Q1 es cerrado, el diodo D3 reacciona y se abre. 2) Para el semiciclo negativo debe operar Q2, de tal manera que Q2, D1 y D4 conforman otro convertidor elevador. En la Figura 2, se presentan los circuitos equivalentes del convertidor AC/DC para los estados de conmutación (interruptor Q1 cerrado (figura 2a), interruptor Q1 abierto y diodo D3 cerrado (Figura 2b)) para el semiciclo positivo del voltaje AC.

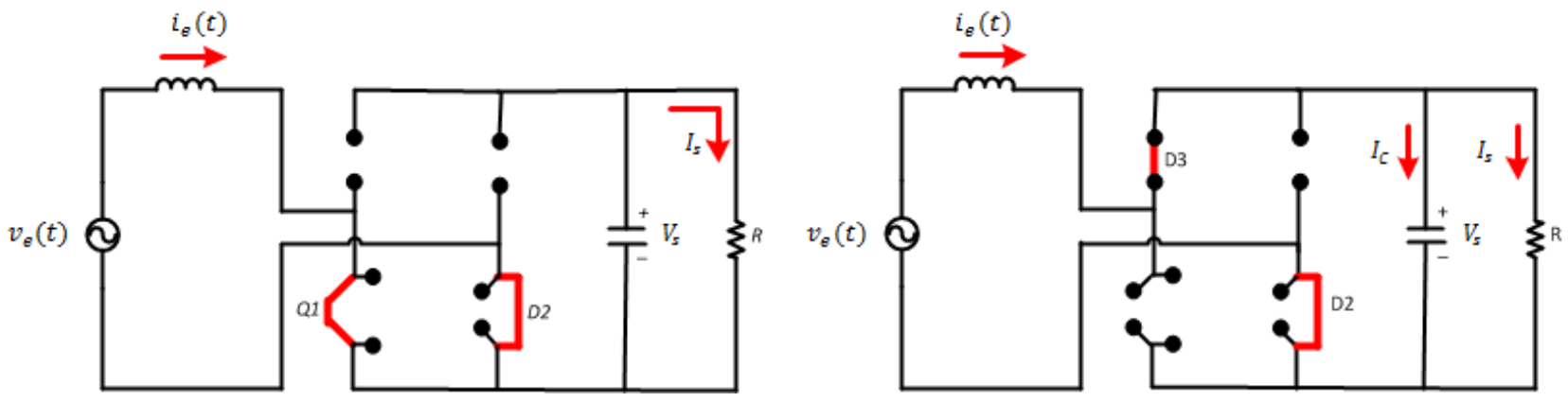

Fig 2. Estados de funcionamiento convertidor AC/DC a) interruptor cerrado, b) interruptor abierto.

En el estado de la figura 2a, al cerrar el interruptor Q1, se crea una malla que involucra: la fuente AC, la inductancia L, el interruptor Q1 y el diodo D2. Asumiendo los semiconductores como interruptores ideales, se tiene a la bobina $L$ queda conectada en paralelo con la fuente $A C$ y por lo tanto su corriente aumentará. En el estado de la figura 2b, el interruptor Q1 se abre y la corriente almacenada en el inductor fluye a través del diodo D1 hacia la carga, aumentando el voltaje en el condensador C. La corriente en el inductor disminuye. El análisis es similar para el semiciclo negativo pero conmutando el interruptor Q2. Nótese que los interruptores Q1 y Q2 deben conmutar a una velocidad mucho mayor que la frecuencia de línea AC, con el fin de controlar el aumento o disminución de la corriente en el inductor y de garantizar una forma de onda senoidal. Por lo tanto, se deben utilizar diodos rápidos para D1 y D2. 


\section{CONTROL PROPUESTO: BALANCE DE POTENCIAS}

Asumiendo que todos los elementos son ideales, la potencia en la fuente de entrada (red AC) y la potencia en la salida del convertidor (red DC) deben ser iguales. A continuación se desarrollaran expresiones para el balance de potencia, las cuales serán utilizadas en el diseño del sistema de control. La ecuación (1) corresponde a la potencia de entrada del convertidor cuando la corriente y la tensión de entrada tienen la misma forma de onda y están en fase, mientras que la ecuación (2) corresponde a la potencia de salida asumiendo rizado cero en la tensión DC del convertidor.

$$
\begin{aligned}
& P_{e}=\frac{1}{T} \int_{0}^{T} v_{e}(t) i_{e}(t) d t=\frac{1}{T} \int_{0}^{T} V_{p} \operatorname{sen}\left(\omega_{0} t\right) \cdot I_{p} \operatorname{sen}\left(\omega_{0} t\right) d t=\frac{V_{p} I_{p}}{2} \\
& P_{S}=V_{s} \cdot I_{s}=\frac{V_{s}^{2}}{R}
\end{aligned}
$$

Donde $V_{p}$ e $I_{p}$ se refieren a los valores pico de las señales de entrada, $\omega_{0}$ a la frecuencia angular fundamental y $T$ es el periodo. Igualando las ecuaciones (1) y (2) se tiene:

$\frac{V_{s}^{2}}{R}=\frac{V_{p} I_{p}}{2}$

El sistema de control tiene como objetivo mantener constante el voltaje DC en la salida, garantizando una buena regulación y el seguimiento del voltaje de referencia elegido por el usuario. Además, el sistema de control también debe garantizar una corriente de entrada de tipo senoidal, con baja distorsión armónica y alto factor de potencia. Las entradas del sistema de control son: los voltajes y las corrientes medidas a la entrada y salida del convertidor $\left(V_{e}, I_{e}, V_{s}, I_{s}\right)$, así como el voltaje de referencia (set-point o valor deseado de voltaje DC en la salida). La salida corresponde a la señal de disparo para los interruptores (figura 3).

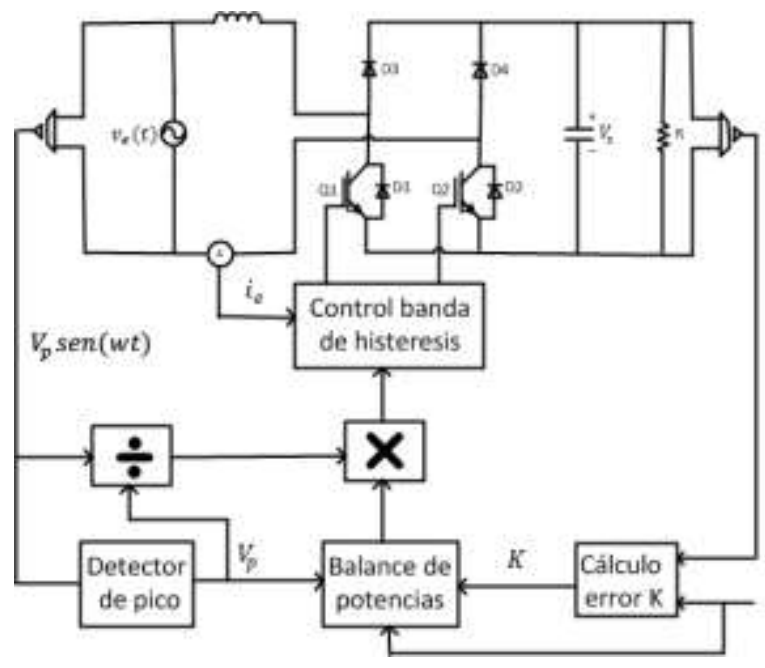

Fig 3. Diagrama del convertidor, indicando entradas y salidas al sistema de control.

Se propone un control digital, con una frecuencia de muestreo mucho mayor a la señal AC (60 Hz). Se ha elegido una frecuencia de muestreo de $40 \mathrm{Khz}$, teniendo en cuenta las limitaciones de los dispositivos de potencia, disparo y control.

Para determinar la fase y la frecuencia del voltaje de entrada se utiliza el siguiente procedimiento: El voltaje es medido a la entrada del convertidor, luego es filtrado digitalmente para obtener su componente fundamental $\left(V_{p} \operatorname{sen}(\omega t)\right)$. es llevado a un detector de pico (también digital), que determina en cada periodo el valor pico $\left(V_{p}\right)$. Luego se realiza la división entre el valor instantáneo de la entrada y el último valor de $V_{p}$ registrado. La salida es una señal seno de magnitud unitaria, que contiene la información de la fase y la frecuencia del voltaje de entrada.

Para obtener la magnitud de la corriente de referencia, se determina el porcentaje de error $(K)$ entre el voltaje de referencia $V_{\text {ref }}$ y el voltaje de salida $V_{s}$, ver ecuación (4). Luego se realiza el balance de potencias de la ecuación (3) y se despeja la corriente $I_{p}$. La corriente $I_{p}$ es usada como la magnitud de la corriente de referencia $\left(I_{\text {ref }}\right)$ para la corriente de entrada del convertidor, ver ecuación (5). La ecuación (5) también tiene 
en cuenta las variaciones del voltaje de salida incluyendo el porcentaje de error $K$. El error $K$ permite disminuir el error en estado estacionario que se produce en el sistema producto de la desviación por la existencia de resistencias parasitas.

$K=\frac{V_{\text {ref }}-V_{s}}{V_{s}}+1$
$\left|I_{\text {ref }}\right|=\frac{2\left[V_{r e f} \cdot K\right]^{2}}{V_{p} R_{L}}$

Para determinar la corriente de referencia en cada instante de tiempo $i_{r e f}(t)$, se multiplican la señal seno unitaria con la ecuación (5). La corriente de entrada $i_{e}(t)$ del convertidor debe seguir la corriente $i_{r e f}(t)$. El control se realiza abriendo y cerrando los interruptores Q1 y Q2 implementando un control de banda de histéresis y siguiendo la secuencia de conmutación establecida en la sección que incluye el principio de funcionamiento del convertidor AC/DC.

\section{IMPLEMENTACIÓN DEL CONVERTIDOR Y RESULTADOS EXPERIMENTALES}

Para la validación del sistema de control propuesto se diseñó y construyó un convertidor elevador AC/DC con PFC (figura 4), siguiendo la topología sin puente de la figura 1. El convertidor es alimentado desde la red $A C(120$ Vrms y $60 \mathrm{~Hz}$ ) y entrega un voltaje de salida regulado entre 170 VDC y 300 VDC. La potencia de diseño es de $300 \mathrm{~W}$.

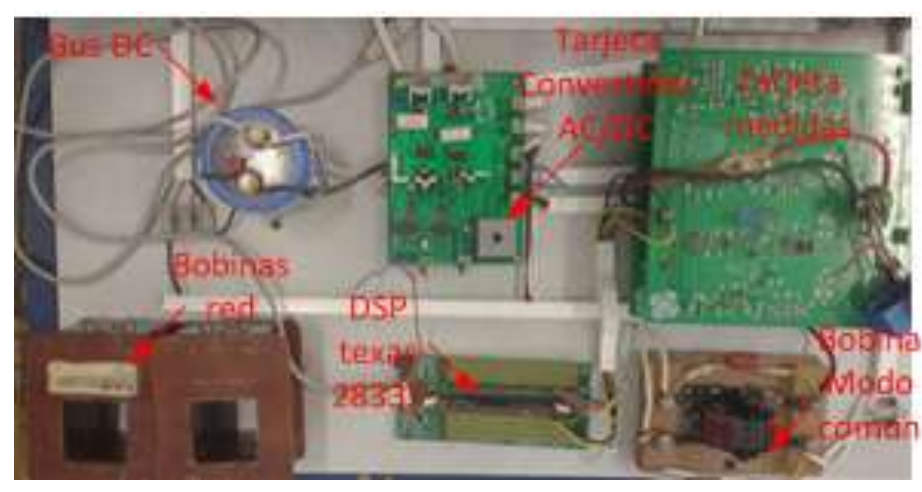

Fig 4. Prototipo convertidor AC/DC implementado.

Se utilizaron transistores de potencia tipo IGBT (IRG4PC40FD de International Rectifier). Estos transistores garantizan un apropiado funcionamiento para las condiciones de frecuencia de conmutación, voltaje y corriente del sistema. Para un correcto funcionamiento de los transistores IGBT, es necesario un circuito manejador de compuerta o driver de disparo. Este circuito fue implementado por medio del circuito integrado ISO5500 (Texas Instruments), el cual a su vez proporciona aislamiento entre los transistores y los elementos de control. Con el fin de controlar los sobrepicos transitorios, debido al proceso de conmutación, y mejorar la compatibilidad electromagnética del sistema, se han implementado redes de snubber en cada uno de los interruptores del sistema (Mejía et al., 2015). Se incluye una bobina choke o bobina de modo común para reducir el ruido de modo común propio de la topología implementada. Los diodos seleccionados son de referencia LXA20T600, estos diodos deben conmutar a la frecuencia impuesta por los IGBTs Q1 y Q2, por lo tanto se trata de diodos para conmutación rápida.

Los valores de la inductancia de entrada del convertidor $(L=10 \mathrm{mH})$ y capacitancia del bus DC $(C=$ $2200 \mu \mathrm{f}$ ) han sido calculados para minimizar el rizado en voltaje y en corriente a las condiciones de carga y frecuencia de conmutación especificadas. Para el esquema de control elegido, no existe una frecuencia de conmutación preestablecida. Solo se puede determinar un límite máximo, equivalente a la mitad de la frecuencia de muestreo del sistema digital de control. Dado que el rizado depende inversamente de la frecuencia de conmutación, no se puede utilizar la frecuencia de muestro para estimar el rizado de peor caso. Se asumió que la frecuencia de conmutación mínima generada por el sistema de control es un tercio de la frecuencia de muestreo del controlador. La medida de los voltajes se realizó utilizando sensores LEM LV-25P. La medida de las corrientes utilizando sensores LEM LAH-50P. Los sensores son basados en efecto hall, brindando aislamiento entre las etapas de potencia y medida. El acondicionamiento de la señal consiste en sucesivas etapas de filtrado, amplificación y offset con el fin de permitir su medida por medio de un DSP TMS320F28335 de Texas Instruments. 
El software PSIM (Powersim Tech co.) permite la simulación de circuitos de electrónica de potencia y la generación de código en C compatible con el DSP TMS320F28335 (Kamiriski et al., 2004; Brijesh et al., 2013). PSIM fue utilizado para la simulación del comportamiento del convertidor con el sistema de control descrito. Para ello se creó el esquemático del circuito físico del convertidor y se agregó un bloque de código C que implementa el algoritmo de control descrito y las respectivas interfaces entre ambos sistemas (sensores de corriente/tensión, acondicionamiento de señales, convertidores ADC, señales digitales de entrada/salida). Una vez verificado el correcto funcionamiento del algoritmo de control se procedió a su implementación en el DSP TMS320F28335. Para esto se utiliza la opción de generación de código fuente de PSIM. Con esto se obtiene un código fuente $C$, conteniendo el algoritmo de control y las rutinas necesarias para el control de todos los periféricos requeridos del DSP. Se utiliza el Code Composer Studio V6 para la compilación del mismo y su posterior programación en la memoria del procesador.

La figura 5 muestra el voltaje en el bus DC, el voltaje de entrada y la corriente de entrada del convertidor. $f p=0.48, V_{e}=172 \mathrm{Vrms}, I_{e}=1.46 \mathrm{Arms}, \mathrm{THD}_{i e}=182 \%, T H D_{v e}=1.9 \%$

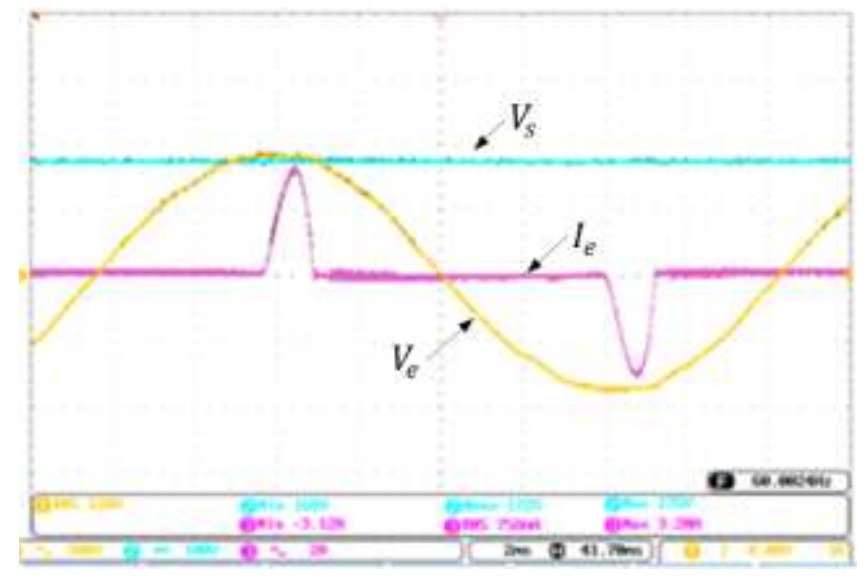

Fig 5. Formas de onda de los voltajes y las corrientes del convertidor.

A continuación se ilustran los resultados obtenidos con el convertidor que incluye la corrección del factor de potencia. Se muestra las pruebas del convertidor a su carga nominal (300 W). La figura 6a. muestra el voltaje y la corriente en la salida del convertidor $\left(V_{s}=299 \mathrm{~V}, I_{s}=0.927 \mathrm{~A}, P_{s}=277.2 \mathrm{~W}\right)$. La figura $6 \mathrm{~b}$. muestra la corriente y la tensión en la entrada del convertidor, la corriente en la entrada es senoidal y está en fase con el voltaje de entrada $\left(V_{e}=119 \mathrm{~V}, I_{e}=2.45 \mathrm{~A}, f p=0.98, P_{e}=291.5 \mathrm{~W}\right)$. La eficiencia es del 95 $\%$ y el THD de corriente es del $15 \%$ (EI THD puede ser mejorado si se instalan filtros en la entrada del convertidor). $V_{e}$

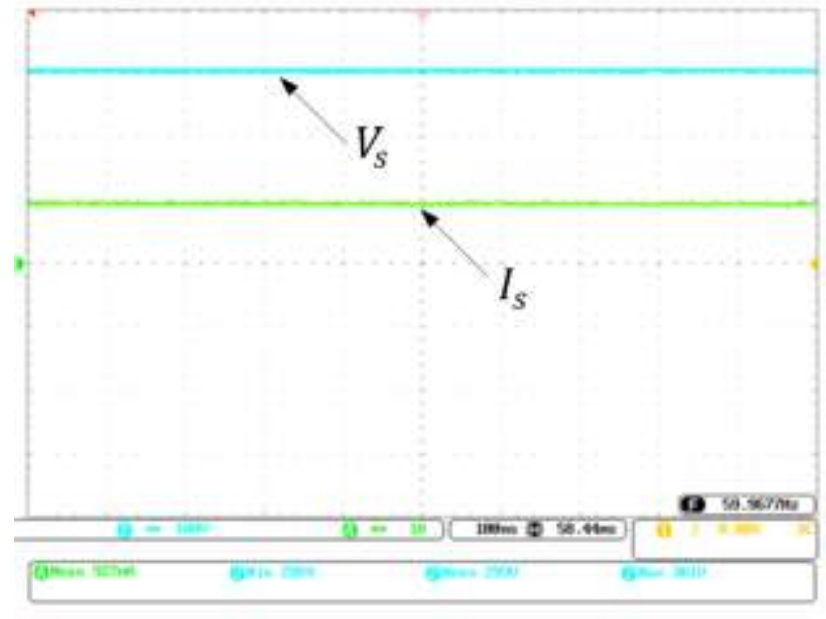

a. Voltaje y corriente salida del convertidor

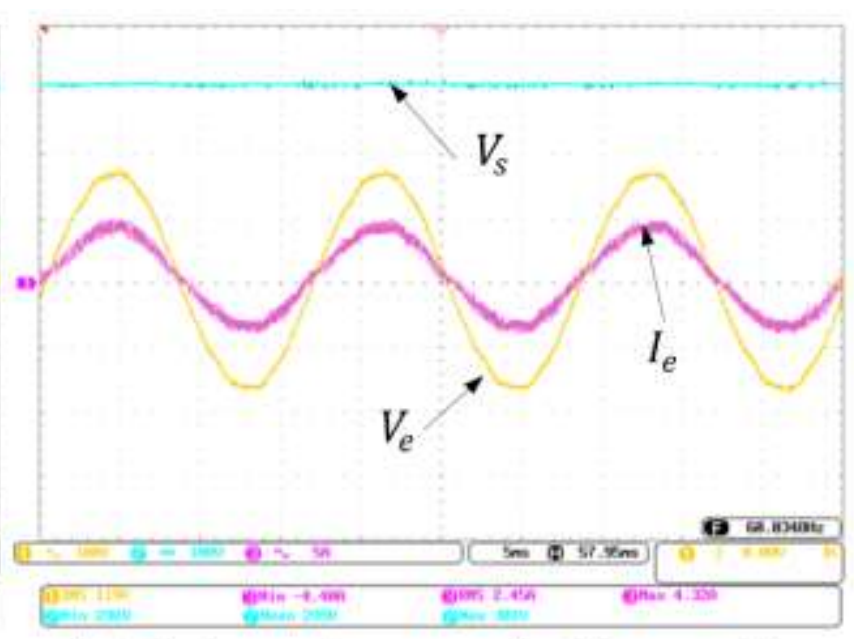

b. Voltaje y corriente entrada del convertidor

Fig 6. Formas de onda de los voltajes y las corrientes del convertidor.

La figura 7a muestra las formas de onda $V_{e}, V_{s}$ e $I_{e}$ cuando se disminuye la corriente de carga del convertidor. Para este caso: 1) $V_{e}$ impuesto por la red permanece constante; 2) $V_{s}$ aumenta momentáneamente cuando se disminuye la corriente de carga pero vuelve a su valor de referencia (200 V) en $680 \mathrm{~ms}$ aproximadamente; 3) $I_{e}$ disminuye de $3.7 \mathrm{~A}$ a 2 A para garantizar el balance de potencias entre 
la entrada y la salida; y 4) La potencia en la entrada y la salida permanecen prácticamente iguales antes ( $\mathrm{P}=$ $440 \mathrm{~W})$ y después $(\mathrm{P}=238 \mathrm{~W})$ de la disminución de la corriente de la carga.

La figura $7 \mathrm{~b}$ muestra las formas de onda $V_{e}, V_{s}$ e $I_{e}$ cuando el voltaje de referencia para $V_{s}$ es aumentado de $170 \mathrm{~V}$ a $200 \mathrm{~V}$. Para este caso: 1) $V_{e}$ impuesto por la red permanece constante; 2) $V_{s}$ efectivamente aumenta de $170 \mathrm{~V}$ a $200 \mathrm{~V}$ siguiendo la referencias de voltaje, esto lo hace en $610 \mathrm{~ms}$; 3) $I_{e}$ aumente de $2.55 \mathrm{~A}$ a 3.7 A, garantizando el balance de potencias entre la entrada y la salida; y 4) La potencia en la entrada y la salida permanecen prácticamente iguales antes $(P=285.6 \mathrm{~W})$ y después $(P=414.4 \mathrm{~W})$ del cambio de referencia de voltaje.

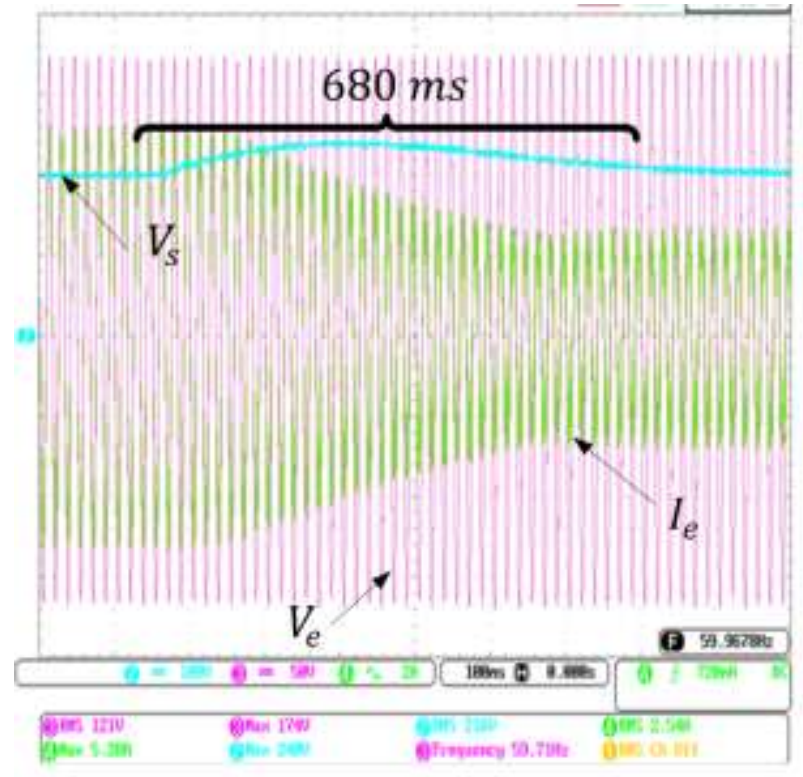

a. Respuesta ante el cambio de carga

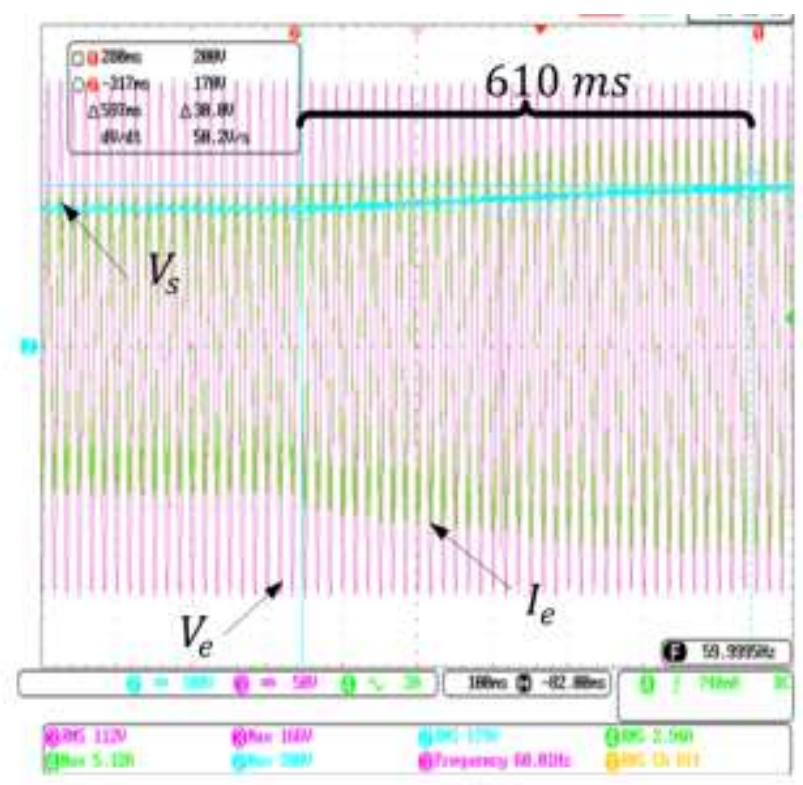

b. Respuesta ante el cambio de voltaje de salida

Fig 7. Respuesta ante el cambio de carga y voltaje en la salida.

\section{CONCLUSIONES}

Se ha logrado diseñar un convertidor AC/DC que permite elevar la tensión de entrada a un valor de referencia programable manteniendo un alto factor de potencia a la entrada. Los resultados obtenidos muestran valores de factor de potencia superiores a 0.95 , distorsión armónica de la onda de corriente menor al $15 \%$ y una alta eficiencia (>90 \%). Se presentaron resultados que incluyen la variación de la corriente de carga y la variación de la tensión de salida, el controlador funcionó apropiadamente ante los cambios con tiempos de respuesta de $680 \mathrm{~ms}$.

El esquema de control implementado se basa en el balance de potencia entre las potencias de entrada y salida del convertidor. El balance de potencias permite obtener la amplitud de la corriente de referencia que permite fijar la tensión del bus DC del convertidor. El control se basa en las condiciones promedio para cada periodo de la señal AC. Esto permite una mayor facilidad de diseño e implementación, permitiendo el uso de sistemas digitales más económicos y un menor tiempo de desarrollo.

Para corregir el error en estado estacionario se propone el uso del error $K$. El error $K$ corrige las desviaciones producidas en la tensión de salida del convertidor ocasionadas por las resistencias parasitas del sistema. Las resistencias parasitas alteran el balance de potencia y podrían ser consideradas en trabajos futuros para obtener la referencia de corriente con mayor precisión.

El sistema de control implementado no genera frecuencias de conmutación constantes. Esta desventaja complica el dimensionamiento del sistema, la predicción de los rizados de corriente y voltaje y el contenido armónico de dichas señales. Como trabajo futuro, se propone la actualización del seguidor de corriente de referencia (comparador) a un controlador P o PI que controle el ciclo de trabajo de una señal PWM y que también este basado en el balance de potencias para solo sintonizar un controlador.

\section{AGRADECIMIENTOS}

Los autores agradecen a la Universidad de Antioquia (UdeA) por el apoyo del programa de sostenibilidad "SOS 2014-2015" 


\section{REFERENCIAS}

Brijesh M. Patel,Kalpesh J.Chudasma, Hardik A.Shah. Simulation of Single Phase Inverter using PSIM Software for Solar P.V. System give Constant Output Voltage at Different Solar Radiation. International Journal of Emerging Trends in Electrical and Electronics (IJETEE), 4(1), (2013)

Das, M. P., Drobnik, J., Jain, P. K., Bakhshai, A., A ZVS Interleaved Boost AC/DC Converter Used in Plug-in Electric Vehicles, Power Electronics, IEEE Transactions on, 27(8), 3513-3529, (2012)

Jovanovic, M. M., Jang, Y., State-of-the-art, single-phase, active power-factor-correction techniques for highpower applications - an overview, Industrial Electronics, IEEE Transactions on, 52(3), 701-708, (2005)

Kamiriski, B.; Wejrzanowski, K.; Koczara, W., An application of PSIM simulation software for rapid prototyping of DSP based power electronics control systems. IEEE Power Electronics Specialists Conference, 1(1), 336-341, (2004)

Lim, J.-W., Kwon, B.-H., A power-factor controller for single-phase PWM rectifiers, Industrial Electronics, IEEE Transactions on, 46(5), 1035-1037, (1999)

Lin, J. L., Yao, W. K. Yang, S. P., Analysis and Design for a Novel Single-Stage High Power Factor Correction Diagonal Half-Bridge Forward AC-DC Converter, Circuits and Systems, IEEE Transactions on, 53(10), 2274-2286, (2006)

Mejía, G. E., Muñoz, N, Cano, J. B., Procedimiento Novedoso para el Diseño del circuito amortiguador (Snubber RCD) y Deducción Matemática de las Ecuaciones, Información tecnológica, 26(2), 117-128, (2015)

Mitchell, D. M., AC-DC Converter Having an Improved Power Factor, U.S. Patent US4412277 A, 25 out. (1983)

Montano, J. C., Salmeron, P., Thomas, J. P., Analysis of power losses for instantaneous compensation of three-phase four-wire systems, Power Electronics, IEEE Transactions on, 20(1), 901-907, (2005)

Muñoz, N., Villada, F., Lopez, J. M., Reducción Selectiva de las Corrientes de Neutro en Instalaciones Eléctricas Mediante el Uso de Compensadores Activos Paralelo, Información Tecnológica, 24(2), 67-78, (2013).

Musavi, F., Edington, M., Eberle, W., Dunford, W. G., Evaluation and Efficiency Comparison of Front End AC-DC Plug-in Hybrid Charger Topologies, Smart Grid, IEEE Transactions on, 3(1), 413-421, (2012)

Qin H., Kimball, J. W., Closed-Loop Control of DC-DC Dual-Active-Bridge Converters Driving Single-Phase Inverters, Power Electronics, IEEE Transactions on, 29(2), 1006-1017, (2014)

Tsang, K.M., Chan, W.L., Adaptive control of power factor correction converter using nonlinear system identification, Power Appl, IEE Proc.-Electr., 152(3), 626-633, (2005)

Zhang, Z., Wang, F., Sun, T., Rodriguez, J., Kennel, R., FPGA-Based Experimental Investigation of a QuasiCentralized Model Predictive Control for Back-to-Back Converters, Power Electronics, IEEE Transactions on, 31(1), 662-674, (2016)

Zielichowski, M., Fulczvk, M., Influence of load on operating conditions of third harmonic ground-fault protection system of unit connected generators, Generation, Transmission and Distribution, IEE Proceedings, 146(3), 241-248, (1999) 